\title{
Experimental Study of Pulse Recovery From Dropout in an Actively Mode-Locked Fiber Laser
}

\author{
Avi Zeitouny and Moshe Horowitz
}

\begin{abstract}
The authors study experimentally the recovery of an actively harmonically mode-locked fiber laser from pulse dropout. Four pulses out of a pulse train of 640 pulses were dropped. The recovery of the pulses that were dropped was studied over a time period of up to $32 \mathrm{~ms}$ with a time resolution on the order of a few picoseconds. The authors show that in the optimal operating region, pulses that are dropped can quickly regenerate. However, when the laser power is insufficient, the probability of a pulse that was dropped to recover is small. When the laser power is high enough, the authors experimentally observed the generation of a pulse pair in a single time slot. The experimental results are in a good quantitative agreement with a comprehensive numerical simulation of the laser.
\end{abstract}

Index Terms-Optical fiber lasers.

\section{INTRODUCTION}

A CTIVELY mode-locked fiber lasers can generate highrepetition-rate short pulses with a very low jitter [1], [2]. Since a fiber laser has a long cavity, the laser is harmonically mode-locked in order to obtain pulses with a high repetition rate. In such an operation mode, some of the cavity pulses may be dropped due to changes in environmental conditions [1], [3]-[5]. This effect limits the laser performance and may limit its use in optical samplers and in optical communication systems.

The operation of actively mode-locked fiber lasers as a function of the power inside the cavity was theoretically studied in [5]. It was found that an actively mode-locked fiber laser passes through four different operating regimes as the intracavity power increases. In the first regime, the intracavity power is small and the pulse amplitude fluctuates in time. In the second regime, the Kerr effect becomes important as the intracavity power increases, leading to pulse shortening and to pulse dropout. When the power further increases, the laser enters a third regime where a stable mode of operation is obtained with very few pulse dropouts. The pulse dropout ratio in this operating regime can be as low as $10^{-12}$ [1]. The laser can quickly recover from accidental pulse dropout only when the laser power increases above a certain threshold that is higher than the threshold of the third operating regime. This subregime is the optimal operating regime of the laser. Finally, it was predicted theoretically that when the power becomes high enough, the

Manuscript received October 8, 2005. This work was supported by the Israel Science Foundation (ISF) of the Israeli Academy of Sciences.

A. Zeitouny is with Elbit Systems, Ltd., Haifa 31053, Israel (e-mail: avize@elbit.co.il).

M. Horowitz is with the Department of Electrical Engineering, TechnionIsrael Institute of Technology, Haifa 32000, Israel (e-mail: horowitz@ee. technion.ac.il).

Digital Object Identifier 10.1109/JLT.2006.881836 laser generates pulse pairs in some of the time slots. The first three operating regimes were observed experimentally [5].

In practical lasers, pulses may drop due to changes in environmental conditions. Pulses that drop should quickly regenerate in order to maintain a reliable operation. We have recently studied theoretically the boundaries of the stable operating regime of an actively mode-locked fiber laser using soliton perturbation theory [6]. In the stable operating regime, the noise around the pulse is stable but becomes unstable when the pulse is dropped, allowing pulse regeneration. We showed that the interaction of the continuum with the modulator and the filter causes energy transfer from the continuum to the pulses. Since solitonlike pulses are stable, the transfer of the energy to the pulses stabilizes the continuum. This effect does not occur in a time slot where a pulse is dropped, and therefore, the noise in such a time slot becomes unstable, and a new pulse can quickly regenerate.

In this paper, we directly study the regeneration of pulses in an actively mode-locked fiber laser after only four pulses are dropped out of a train of 640 pulses. The regeneration of the specific pulses that are dropped is measured with a time resolution on the order of picoseconds over a long time scale of up to $32 \mathrm{~ms}$, which is equivalent to 93000 cavity round trips. The result is compared to a comprehensive numerical simulation of the laser. A good quantitative agreement between the theoretical and the experimental results is obtained. We found that the laser could quickly recover from pulse dropout only when the laser power was above a certain threshold. We note that in previous works, pulse dropout was only studied indirectly by performing measurements on the whole pulse train using an RF spectrum analyzer or a sampling oscilloscope [5]. However, such measurements do not give the dynamics of the pulse recovery, and it also does not track the buildup of a specific pulse. We also note that in a previous work, an experimental study of timing restoration dynamics of an actively mode-locked fiber laser was studied [7]. However, in this paper, a perturbation was applied to the entire pulse train and the laser dynamics was studied indirectly by measuring the phase of the entire train of pulses. We also describe in this paper, experimental results that demonstrate the generation of pulse pairs in a single time slot when an actively harmonically mode-locked fiber laser is excessively pumped. This effect was predicted theoretically [5]; however, to the best of our knowledge, the experimental observation of this effect has yet to be reported.

\section{EXPERIMENTAL SETUP}

The laser configuration used in our experiments is shown in Fig. 1. The laser is based on the ring configuration and is 


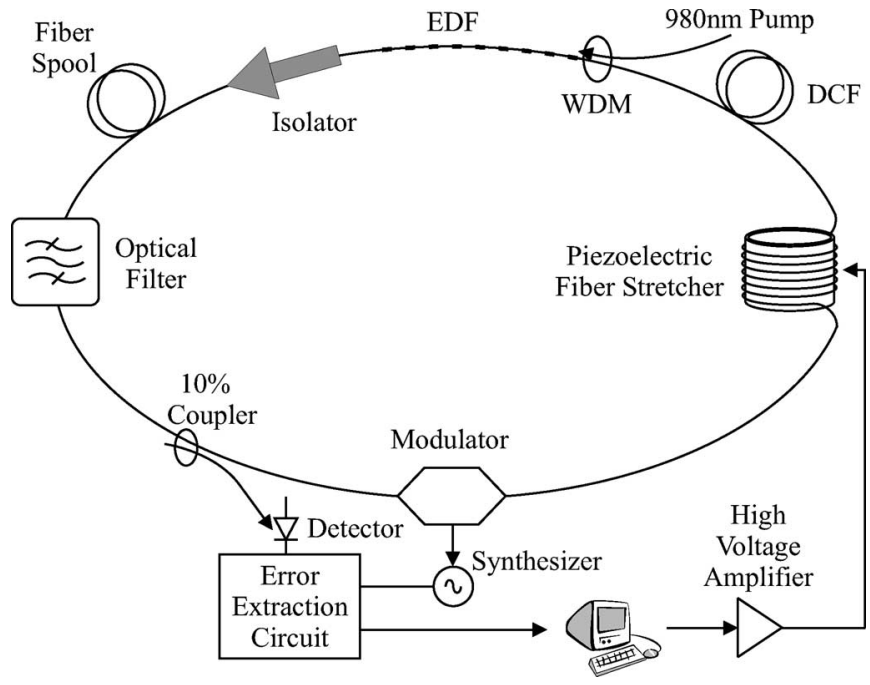

Fig. 1. Schematic description of the fiber laser used in the experiment. DCF is a dispersion-compensating fiber, EDF is an erbium-doped fiber, and wavelength division multiplexing (WDM) is a coupler used to pump the EDF using a 980-nm laser. All the fibers and the components in the laser are polarization maintaining.

built entirely of polarization maintaining fiber-components. Active mode locking is obtained using a Mach-Zehnder modulator. The length of the cavity was about $66 \mathrm{~m}$, and therefore, at a modulation frequency of $2 \mathrm{GHz}, 640$ pulses simultaneously propagated in the cavity. The modulation depth of the modulator was close to 1 . The cavity loss of the laser was approximately $10 \mathrm{~dB}$. An optical filter with a bandwidth of $14 \mathrm{~nm}$ was used to control the laser wavelength. A dispersion compensating fiber (DCF) with a length of $7 \mathrm{~m}$ and a dispersion coefficient of $-40 \mathrm{ps} / \mathrm{nm} \cdot \mathrm{km}$ was used in order to lower the average dispersion of the cavity. The average dispersion of the laser was estimated to be about $3.8 \mathrm{ps} / \mathrm{nm} \cdot \mathrm{km}$. The average dispersion was also measured by tuning the optical filter in the cavity and measuring the frequency change in the cavity modes spacing using an radio frequency (RF) spectrum analyzer. The relation between the change in the optical wavelength $\Delta \lambda$ and the change in the frequency of the cavity mode $\Delta f_{\mathrm{M}}$ is given by

$$
\frac{\Delta f_{\mathrm{M}}}{\Delta \lambda}=-c f_{\mathrm{M}} D
$$

where $c$ is the speed of light, $f_{\mathrm{M}}$ is the modulation frequency, and $D$ is the average dispersion. A stable operation was obtained when the average laser power at the input of the coupler was about $3 \mathrm{~mW}$. Under these conditions, the pulses had a full-width at half-maximum (FWHM) bandwidth of $0.9 \mathrm{~nm}$ and had a pulse duration, measured using an autocorrelator, of about 3 ps, assuming a secant-hyperbolic pulse shape. The DCF was put in the laser described in this paper, at the input of the amplifier and therefore the nonlinear effect in this fiber was significantly smaller than in the laser described in [5]. Therefore, the measured autocorrelation trace was close to the autocorrelation trace of a secant-hyperbolic pulse rather than a Gaussian pulse, as described in [5]. Fluctuations in the length of the cavity, caused by temperature changes and vibrations,

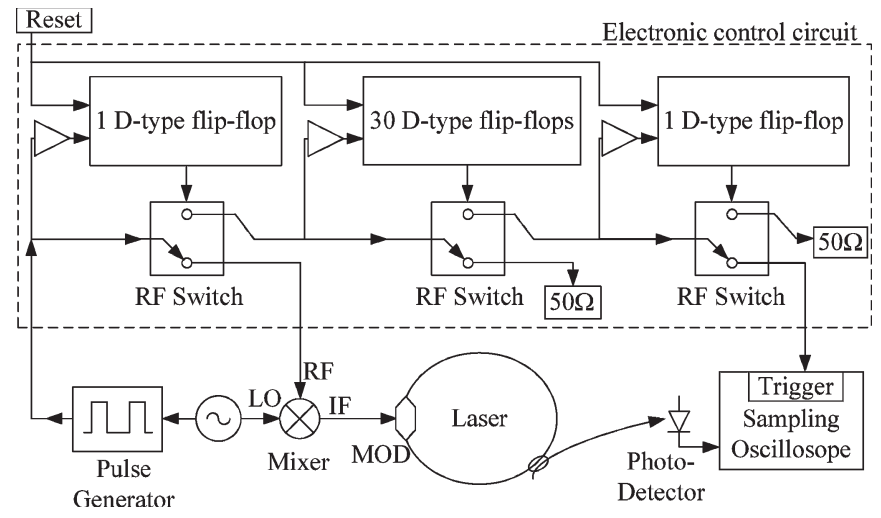

Fig. 2. Schematic description of the experimental setup used to drop pulses in the laser and to obtain a long delay needed to track the dropped pulses. MOD is a Mach-Zehnder electrooptical modulator used for the active mode locking of the laser.

were compensated by an electrooptic feedback loop, based on a fiber rotated on a piezoelectric tube. The phase of the pulse train at the output of the laser was compared with the phase of the electrical synthesizer used to drive the modulator by using an electrical mixer. The signal at the output of the mixer was passed through a low-pass filter and was sampled by a computer. The computer software realized a proportionalintegral-derivative controller [8] that was connected to a highvoltage amplifier that drove the piezoelectric fiber stretcher. The response time of the electronic feedback was about $10 \mathrm{~ms}$.

In our experiment, pulses were dropped by controlling the voltage to the electrooptic modulator used for the active mode locking of the laser. The measurement of the pulses was performed using a sampling oscilloscope. The electronic circuit used to drop the pulses and to add the delay required to track the dropped pulses is shown in Fig. 2. We used an electrical pulse generator (HP 8133A) for dropping the pulses in the laser and for triggering the sampling oscilloscope. This device generated square pulses that were synchronized to the signal generator that drove the modulator. The pulse generator could be programmed to repeatedly generate sequences of 32 programmable pulses followed by up to 15000 sequences of 32 zero bits. We used this mode of operation in the experiment to obtain long delays, as described below.

The most difficult task in our experiment was to accurately track the recovery of the dropped pulses over a very long time delay. The tracking of a particular pulse required to measure the pulse over a time scale of up to about $32 \mathrm{~ms}$ with an accuracy on the order of a few picoseconds. The very long time delay versus the need for a very high accuracy and a very high time resolution, as required in our experiment, could not been achieved directly from our sampling oscilloscope (Agilent 81600B). Therefore, we designed the electrical control circuit marked by the dashed box in Fig. 2. The delay required to track the dropped pulses was obtained using a cascade of RF switches, controlled by a latch circuit. The electrical pulses of the pulse generator functioned as the clock of the D-type flip-flops that were used to realize the latching operation and changed their position. Assuming that the output of the D-type flip-flop is in the zero state, a clock pulse will change the output of the flip-flop, since their input port was connected to a constant 
bias. Additional clock pulses do not change the output of the flip-flop. When $n$ D-type flip-flops were cascaded, the output of the $n$th flip-flop will change only after $n$ clock pulses.

The configuration of the switches, described in Fig. 2, shows the initial position of the switches before the pulses were dropped. The first electrical pulse that entered the circuit was passed toward the modulator. The control of the modulator loss by the electrical pulses was performed by mixing the square electrical pulses, generated by the electrical pulse generator, with the sinusoidal wave that drove the modulator using a double balanced mixer. Without supplying the electrical pulses, the loss in the modulator was minimum when the optical pulses passed through the modulator. When the electrical pulses were present, the phase of the sinusoidal wave at the input of the modulator was inverted by $180^{\circ}$. Therefore, when an electrical pulse was supplied, the transmission of the modulator was inverted, and the optical pulses that passed at that time in the modulator were blocked.

The circuit described above can be used to drop and to track only a single pulse out of the pulse train. In our experiment, we were restricted by the speed of the D-type flip-flops. The flip-flops used in our experiment required a control pulse with a minimum duration of about $2 \mathrm{~ns}$. This minimum electrical pulse duration was four times wider than the period of the laser pulses. Therefore, in our experiment, about four pulses out of a train of 640 pulses were dropped.

The RF switches and the D-type flip-flops were also used to obtain the long delay required to track the dropped pulses. The first pulse changed the state of the first RF switch and caused the dropout of four pulses in the laser. The next 30 electrical pulses changed the position of 30 cascaded D-type flip-flops and then changed the position of the second RF switch. The 32nd pulse was routed to the trigger input of the sampling oscilloscope and changed the position of the third RF switch. The rest of the pulses were fed into a matched $50-\Omega$ termination. Therefore, an entire train of electrical pulses caused only a single dropout event in the laser and triggered the sampling oscilloscope only once. In order to measure the whole pulse trace, the switches and the electrical pulse generator were reset, and the measurement was repeated. The short time delay needed to obtain a single pulse trace at a specific delay was controlled by the sampling scope. In order to obtain a pulse trace after a long time delay a different periodicity between the electrical pulses of the generator was chosen. The periodicity was adjusted by changing the number of zeros that follow each electrical pulse. Since the pulse generator was synchronized to the synthesizer used to derive the optical modulator, high accuracy in the measurement was obtained, even after a very long time delay. The maximum time delay provided by the control of the pulse generator and the electronic circuit was about $8 \mathrm{~ms}$. In order to increase the maximum delay to $32 \mathrm{~ms}$, we divided the frequency of the signal used to synchronize the pulse generator by four.

\section{RESUlTS AND DISCUSSION}

In order to measure the ability of the laser to recover from pulse dropout, the measurements were performed at two power

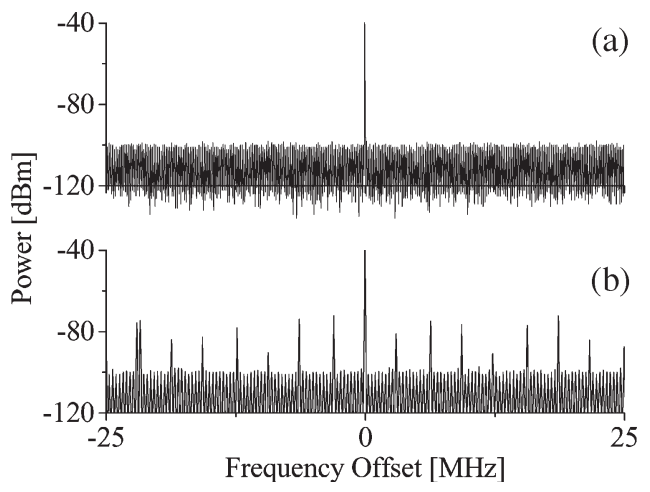

Fig. 3. (a) Electrical spectrum of the laser pulses around a frequency of $2 \mathrm{GHz}$ for an average laser power of $3.0 \mathrm{~mW}$ where supermodes are suppressed by $60 \mathrm{~dB}$ compared to the main mode and (b) for an average laser power of $2.5 \mathrm{~mW}$, where supermodes are clearly observed due to pulse dropouts.

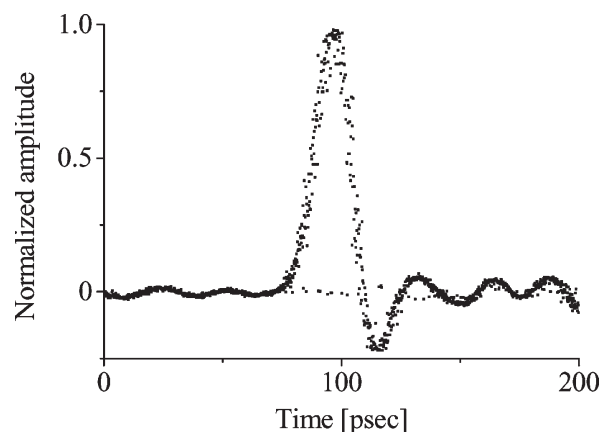

Fig. 4. Pulse profile, measured using a sampling oscilloscope. The flat line at the bottom of the pulse profile indicates that some of the pulses are missing. The average laser power at the input of the coupler was $2.5 \mathrm{~mW}$.

levels, above and below the threshold needed to enable fast recovery from pulse dropout. We used an RF spectrum analyzer and a sampling oscilloscope to identify a significant amount of dropout events in the laser. The repetition frequency of the laser pulses was $2 \mathrm{GHz}$. Fig. 3 shows the RF spectrum, measured around a frequency of $2 \mathrm{GHz}$, when the intracavity laser power was approximately 3 and $2.5 \mathrm{~mW}$. When the power is insufficient, pulse dropout causes a significant increase in the level of the supermodes, as can be observed in Fig. 3(b). When the cavity power is high enough the amount of pulse dropout is negligible, as can be observed in Fig. 3(a), where the supermodes are suppressed by about $60 \mathrm{~dB}$ in comparison to the level of the main mode. The existence of pulse dropouts at the lower power level can also be observed by the corresponding sampling oscilloscope trace shown in Fig. 4. The line at the bottom of the trace indicates that some of the time slots were empty. This line did not exist when the laser was operated in the optimal operating region. Fig. 4 also indicates that although many pulses were dropped, the pulses that propagated in the cavity were stable and had a low noise. Fig. 4 can be used to estimate the number of pulses that were missing in the pulse train. From the ratio between the number of points that correspond to pulses that were dropped and the number of points that correspond to pulses that propagated in the cavity, calculated at the first 50-ps interval after the beginning of the pulse, we can estimate from Fig. 4 that about $10 \%$ of the pulses were missing in the pulse train. 


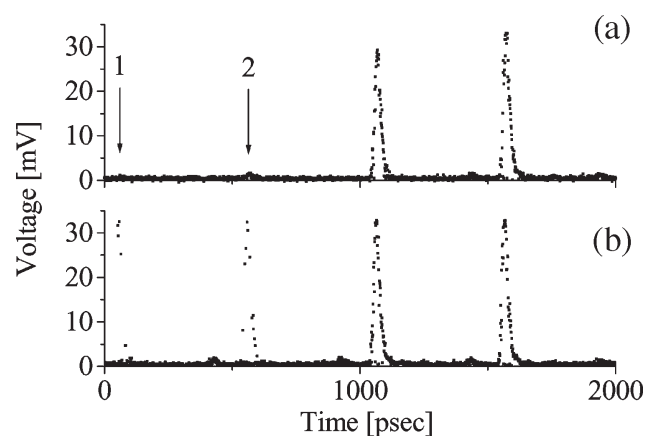

Fig. 5. Pulse traces measured using a sampling oscilloscope and the control circuit shown in Fig. 2 at a power level below the threshold needed to recover from pulse dropout, (a) 31 round trips and (b) 96000 round trips after the first two pulses, marked by 1 and 2 , were dropped. The average laser power at the input of the coupler was $2.5 \mathrm{~mW}$.

The pulse train when the laser power was below the power needed to recover from pulse dropout was measured 31 and 96000 round trips after dropping the pulses. The oscilloscope traces are shown in Fig. 5. The average laser power in this case was about $2.5 \mathrm{~mW}$ at the input of the coupler. The time scale of the oscilloscope was chosen in order to measure four pulses out of the pulse train. Fig. 5(a) shows the sampling oscilloscope traces 31 round trips after the pulses were dropped. The figure shows that the first two pulses, marked by "1" and "2," were almost completely dropped while the other two pulses continued to propagate in the laser cavity. The figure also shows the existence of a line at the button of the pulses that were not intentionally dropped, indicating accidental pulse dropout events, as obtained in Fig. 4. Fig. 5(b) shows the four pulses and 96000 round trips after the dropout of the first two pulses. The figure shows that the pulses could not recover from the dropout in most cases. However, in contrary to the theoretical results [5], the missing pulses did grow in some of the cases. In the simulation given in [5], only a single pulse was dropped out of a pulse train of 10000 pulses when the laser was just at the threshold of optimal operation. Therefore, the affect of the pulse dropout on the amplifier gain was very small. Hence, pulses that were dropped when the laser operated in the second operating region, could regenerate only due to very large spontaneous noise, generated by the amplifier. Since the probability of such an event is very low, pulses almost never regenerated. In our experiment, a significant number of pulses were missing from the pulse train because the laser was operated below threshold. Moreover, four pulses instead of one were dropped out of the remaining pulse train. Therefore, in our experimental setup there is a large fluctuation in the amplifier gain and the probability for a spontaneous recovery from pulse dropout increases in comparison to the theoretical results given in [5]. Fig. 5(b) also indicates that the probability for a spontaneous pulse recovery depends on the amplitude of the pulse after the dropout. The pulse marked by "2" in Fig. 5, which was not completely dropped as the pulse marked by " 1 ," had a higher probability to regenerate. Twelve points in Fig. 5(b) correspond to pulses that recovered after the dropout of pulse " 2 ," while only five points correspond to pulses that recovered after the dropout of pulse "1."

At power levels above threshold, pulses that were dropped can regenerate. Measurements of a single pulse that was

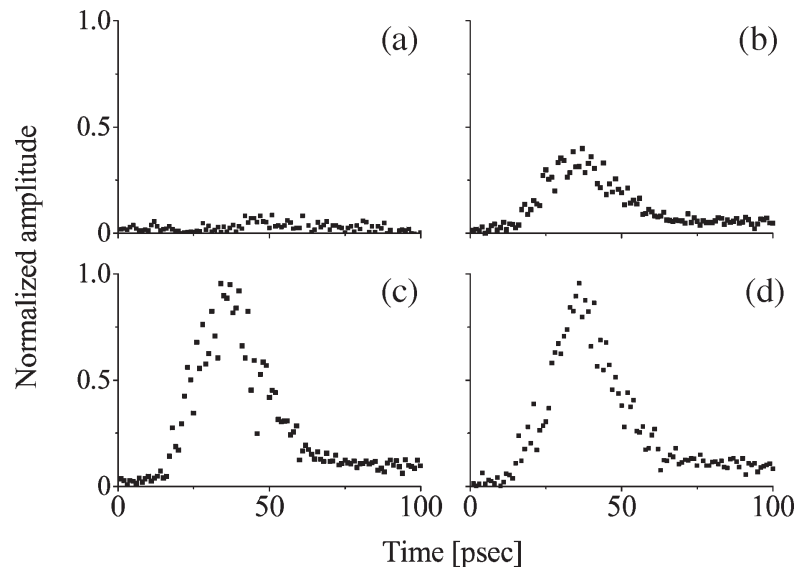

Fig. 6. Pulse traces measured using a sampling oscilloscope and the control circuit shown in Fig. 2 obtained after (a) 38 round trips, (b) 410 round trips, (c) 1061 round trips, and (d) 1712 round trips after the pulse was dropped. The average laser power at the input of the coupler was $3 \mathrm{~mW}$.

dropped were taken at different time delays after the pulse dropout. The average laser power at the input of the coupler was $3 \mathrm{~mW}$. Typical traces measured using the sampling oscilloscope and the control circuit, given in Fig. 2, are shown in Fig. 6. The figure shows that the pulses that were dropped regenerated after about 1700 round trips. The decrease in the pulse duration as the pulse power increases, as predicted by theory [5], cannot be observed in the figure since the pulse duration is on the order of a few picoseconds while the minimum time resolution of the photodetector is about $30 \mathrm{ps}$.

The results obtained in the experiment were compared to a numerical simulation of the laser. The system and the dispersion parameters of the laser were identical to that described in Section II. The simulation was based on the superpulse method [5] in order to simulate the dropout of a limited number of pulses out of a pulse train that contained 640 pulses. A distributed amplifier model with a response time of $160 \mu \mathrm{s}$, which is equivalent to about 500 round trips, was used. The pulse measured in the oscilloscope is equal to the convolution between the intensity pulse profile of the optical pulses $P(t)$ and the electrical impulse response of the measurement system $h(t)$. The impulse response of the measurement system was mainly affected by the photodetector and the sampling oscilloscope response time. The impulse response function had a FWHM of $26 \mathrm{ps}$. Since the duration of the laser pulses was on the order of $3 \mathrm{ps}$, and the response time of the measurement system was on the order of $26 \mathrm{ps}$, we assumed that the laser pulses are significantly shorter than the duration of the impulse response of the measurement system. Therefore, we could write the following relation between the pulse profile measured in the sampling oscilloscope $R(t)$ and the input optical pulse intensity $P(t)$ :

$$
\begin{aligned}
R(t) & =P(t) * h(t)=\int_{-\infty}^{\infty} P(t-\tau) h(\tau) d \tau \\
& \approx h(t) \int_{-\infty}^{\infty} P(t-\tau) d \tau=h(t) \cdot E
\end{aligned}
$$




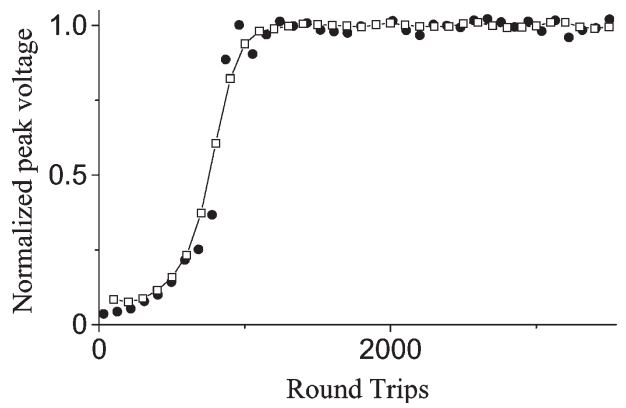

Fig. 7. Comparison between the theoretical (solid line and squares) and the experimental measurement (circles) of the pulse energy, normalized to the energy at steady state, as a function of the number of round trips after the pulse was dropped. The average laser power at the input of the coupler was $3 \mathrm{~mW}$.

where $*$ denotes the convolution operator, and $E$ is the energy of the pulse $E=\int_{-\infty}^{\infty} P(\tau) d \tau$. Equation (2) shows that in our measurement system, the amplitude of the pulse in the sampling oscilloscope is proportional to the energy of the optical pulses in the laser. Therefore, we did not have to measure the exact shape of the electrical impulse response $h(t)$. In order to compare the theoretical and the experimental results, pulse traces, similar to that shown in Fig. 6, were measured after about 40 different delays. Fig. 7 shows the comparison between the time dependence of the optical pulse energy, calculated using the numerical simulation, and the pulse energy, obtained from the pulse traces measured experimentally after different delays. The energy was normalized to the energy of the pulse at steady state. The figure shows that a very good quantitative agreement is obtained between theory and experiment. The figure also indicates that the pulse is rapidly built and then settles down toward the steady-state pulse with some oscillations. We believe that these oscillations are caused due to the dynamics of the erbium-doped fiber amplifier. The measured time needed for a dropped pulse to recover up to $90 \%$ of the steady-state energy was $0.3 \mathrm{~ms}$. The minimum power needed to enable the laser to recover from pulse dropout, as calculated using the numerical simulation, was about $2.8 \mathrm{~mW}$. This result is in accordance with the measured power $-2.7 \mathrm{~mW}$. We believe that the small difference between the theoretical and the experimental power values is caused due to the effect of environmental conditions on the laser operation. Changes in environmental conditions cause pulse dropouts that affect the amplifier gain. The fluctuations in the gain enable the regeneration of pulses at a smaller power than theoretically required, as can also be observed in Fig. 5 .

When the laser power is further increased, theory predicts that a pulse pair will be generated in a single time slot [5]. The separation between the pulses is expected to be smaller than the resolution of the sampling oscilloscope. Therefore, we measured the generation of pulse pairs in the time domain using an autocorrelator. The generation of multiple pulses in a laser cavity was observed in passively mode-locked fiber lasers [9]. However, to the best of our knowledge, the generation of a pulse pair in a single time slot has not yet been experimentally observed in harmonically actively mode-locked fiber lasers. In the measurement, we faced a difficulty to measure a stable autocorrelation trace since pulse pairs might not be generated in all the time slots and since nonlinear interaction between

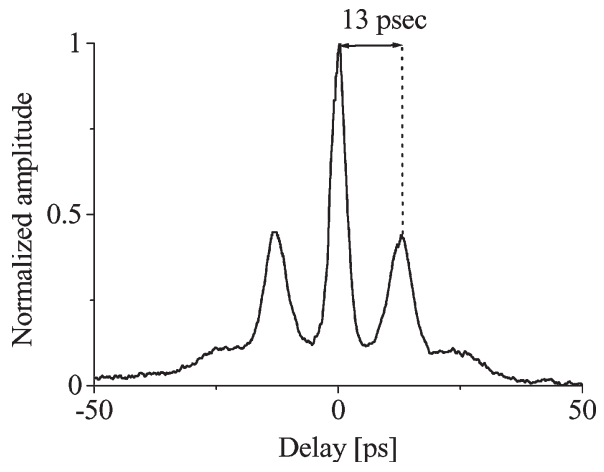

Fig. 8. Autocorrelation trace of the laser pulses at the fourth operating regime. The autocorrelation shows the generation of a pulse pair with a time separation between the pulses of about $13 \mathrm{ps}$. The average laser power was $6 \mathrm{~mW}$.

the two pulses may cause fluctuations in the pulses. Moreover, instability of the pulse train also deteriorates the operation of the electrical control circuit, which is used to stabilize the laser cavity. However, a careful adjustment of the pump power enabled us to obtain steady-state operation, where two pulses existed in almost all the time-slots. The measured intensity autocorrelation trace of the pulse pair is shown in Fig. 8 for an average laser power of $6 \mathrm{~mW}$. The autocorrelation trace indicates that two pulses were generated in a single time slot. The time separation between the pulses was about 13 ps. The width of each pulse was approximately $3.4 \mathrm{ps}$, as calculated from the center peak in the autocorrelation trace. The ratio between the amplitude of the central peak and the amplitudes of the other two peaks in the autocorrelation trace was approximately 0.45 , indicating that the amplitudes and the profiles of the two pulses were similar. The optical spectrum of the laser also contained a sinusoidal modulation that corresponds to the existence of the pulse pair. The modulation in the spectrum implies that the two pulses are mutually coherent. The measured minimum power that was required for the fourth operating regime was $3.3 \mathrm{~mW}$. At this power, an instability in the pulse train was observed in the sampling oscilloscope and in the RF spectrum analyzer. The theoretical boundary for the fourth operating regime, as calculated using the numerical simulation, was $3.1 \mathrm{~mW}$.

\section{CONCLUSION}

We have directly studied, for the first time to our knowledge, the dynamics of pulse recovery in an actively harmonically mode-locked fiber laser after a pulse dropout event occurs. Four pulses out of a pulse train of 640 pulses were dropped. The recovery of the dropped pulses over a time period of more than $30 \mathrm{~ms}$ was directly studied with a time accuracy on the order of a few picoseconds. When the laser is insufficiently pumped, spontaneous dropouts occur and pulses that are dropped have a low probability to regenerate on a time scale of about $30 \mathrm{~ms}$. When the laser is pumped above a certain threshold pulses can quickly recover from dropout. The experimental results were in a good quantitative agreement with a comprehensive numerical simulation of the laser. We also observed the generation of a pulse pair in a single time slot when the laser was excessively pumped. This effect was predicted theoretically; however, it 
has not been confirmed experimentally in harmonically actively mode-locked fiber lasers until now.

\section{ACKNOWLEDGMENT}

The authors would like to thank $M$. Namer and Y. Komorovsky for their assistance in designing the electrical circuit and to O. Levi for her ideas on designing the experimental setup.

\section{REFERENCES}

[1] T. F. Carruthers and I. N. Duling, III, "10-GHz, 1.3-ps erbium fiber laser employing soliton pulse shortening," Opt. Lett., vol. 21, no. 23, pp. 1927-1929, Dec. 1996.

[2] M. E. Grein, H. A. Haus, Y. Chen, and E. P. Ippen, "Quantum-limited timing jitter in actively modelocked lasers," IEEE. J. Quantum Electron., vol. 40, no. 10, pp. 1458-1470, Oct. 2004.

[3] M. Nakazawa, E. Yoshida, and Y. Kimura, "Ultrastable harmonically and regeneratively modelocked polarization-maintaining erbium fiber ring laser," Electron. Lett., vol. 30, no. 19, pp. 1603-1605, Sep. 1994.

[4] X. Shan and D. M. Spirit, "Novel method to suppress noise in harmonically modelocked erbium fiber lasers," Electron. Lett., vol. 29, no. 11, pp. 979-981, May 1993.

[5] M. Horowitz, C. R. Menyuk, T. F. Carruthers, and I. N. Duling, III, "Theoretical and experimental study of harmonically modelocked fiber lasers for optical communication systems," J. Lightw. Technol., vol. 18, no. 11, pp. $1565-1574$, Nov. 2000

[6] A. Zeitouny, Y. Parkhomenko, and M. Horowitz, "Stable operating region in a harmonically-actively mode-locked fiber laser," IEEE J. Quantum Electron., vol. 41, no. 11, pp. 1380-1387, Nov. 2005.
[7] M. E. Grein, L. A. Jiang, Y. Chen, H. A. Haus, and E. P. Ippen, “Timing restoration in an actively mode-locked fiber ring laser," Opt. Lett., vol. 24, no. 23, pp. 1687-1689, Dec. 1999.

[8] G. F. Franklin, J. D. Powell, and A. Emami-Naeini, Feedback Control of Dynamic Systems, 4th ed. Englewood Cliffs, NJ: Prentice-Hall, 2002.

[9] A. Gordon, B. Vodonos, V. Smulakovski, and B. Fischer, "Melting and freezing of light and modes in mode-locked lasers," Opt. Express, vol. 11, no. 25, pp. 3418-3424, Dec. 2003.

Avi Zeitouny was born in Haifa, Israel, in 1971. He received the B.A. degree in physics, the B.Sc. degree in materials engineering, and the M.Sc. degree in materials engineering from Technion-Israel Institute of Technology, Haifa, where he is currently working toward the Ph.D. degree in electrical engineering in 1996 and 1999, respectively.

His research interests include fiber lasers and their applications.

Moshe Horowitz received the Ph.D. degree from Technion-Israel Institute of Technology, Haifa, Israel, in 1994.

Since 1998, he has been a Professor with the Department of Electrical Engineering, Technion. His current research interests include inverse scattering theory in fiber gratings and its applications for developing novel fiber sensors, novel fiber lasers, and microwave photonics. 